\title{
Compositionality Entails Sequentializability
}

\author{
Pranav Garg and P. Madhusudan \\ University of Illinois at Urbana-Champaign
}

\begin{abstract}
We show that any concurrent program that is amenable to compositional reasoning can be effectively translated to a sequential program. More precisely, we give a reduction from the verification problem for concurrent programs against safety specifications to the verification of sequential programs against safety specifications, where the reduction is parameterized by a set of auxiliary variables $A$, such that the concurrent program compositionally satisfies its specification using auxiliary variables $A$ iff the sequentialization satisfies its specification. Existing sequentializations for concurrent programs work only for underapproximations like bounded context-switching, while our sequentialization has the salient feature that it can prove concurrent programs entirely correct, as long as it has a compositional proof. The sequentialization allows us to use sequential verification tools (including deductive verification tools and predicate abstraction tools) to analyze and prove concurrent programs correct. We also report on our experience in the deductive verification of concurrent programs by proving their sequential counterparts using the program verifier BoogIE.
\end{abstract}

Keywords: concurrent programs, compositional verification, sequentialization.

\section{Introduction}

Sequentializing concurrent programs has been a topic of recent research. Given a concurrent program with a safety specification, we would like to reduce the problem of verifying the concurrent program to the verification of a sequential program. Moreover, and most importantly, we seek a sequential program that does not simply simulate the global evolution of the concurrent program as that would be quite complex and involve taking the product of the local statespaces of the processes. Instead, we seek a sequential program that tracks a bounded number of copies of the local and shared variables, where the bound is independent of the number of parallel components.

The appeal of sequentialization is that it allows using the existing class of sequential verification tools to verify concurrent programs. A large number of sequential verification techniques and tools, like deductive verification, abstractionbased model-checking, and static dataflow analysis immediately come into play when a sequentialization is possible.

Of course, such sequentializations are not possible for all concurrent programs and specifications. In fact, in the presence of recursion and when variables have 
bounded domains, concurrent verification is undecidable while sequential verification is decidable, which proves that an effective sequentialization is in general impossible.

The currently known sequentializations have hence focussed on capturing under-approximations of concurrent programs. Lal and Reps [13] showed that given a concurrent program with finitely many threads and a bound $k$, the problem of checking whether the concurrent program is safe on all executions that involve only $k$ context-rounds can be reduced to the verification of a sequential program. A lazy sequentialization for bounded context rounds that ensures that the sequential program explores only states reachable by the concurrent program was defined by La Torre et al [10. A sequentialization for unboundedly many threads and bounded round-robin rounds of context-switching is also known [1]. Lahiri, Qadeer and Rakamarić have used the sequentialization of Lal and Reps to check concurrent C-programs by unrolling loops in the sequential program a bounded number of times, and subjecting them to deductive SMT-solver based verification 128 ].

In this paper, we show a general sequentializability result that is not restricted to under-approximations. We show that any concurrent program with finitely many threads can always be sequentialized provided there exists a compositional proof of correctness of the concurrent program. More precisely, we show that given a concurrent program $C$ with assertions and a set of auxiliary variables $A$, there is a sequentialization of it, $S_{C, A}$ with assertions, and that $C$ can be shown to compositionally satisfy its assertions by exposing the auxiliary variables $A$ if and only if $S_{C, A}$ satisfies its assertions. The notion of $C$ compositionally satisfying its assertions using auxiliary variables $A$ is defined semantically, and intuitively captures the rely-guarantee proofs pioneered by Jones [9]. Rely-guarantee proofs of concurrent programs are very standard, and perhaps the best known compositional verification technique for concurrent programs. In these proofs, auxiliary variables can be seen as local states that get exposed in order to build a compositional rely-guarantee proof.

Compositional proofs of programs may not always exist, and since our sequentialization only produces sequential programs that are precise when a compositional proof exists over the fixed auxiliary variables $A$, proving its sequentialization correct can be seen as a sound but incomplete mechanism for verifying the concurrent program. Note that our sequentialization does not require the compositional proof to be given; it is only parameterized by the auxiliary variables $A$. In fact, if the sequential program is correct, then we show that the concurrent program is always correct. Conversely, if the concurrent program is correct and has a compositional proof using variables $A$, then we show that the sequential program is guaranteed to be correct as well.

The salient aspect of our sequentialization is that it can be used to prove concurrent programs entirely correct, as opposed to checking underapproximations of it. Moreover, though our sequentializations are sound but incomplete, we believe they are useful on most practical applications since concurrent programs often have compositional proofs. Our result also captures the cost of 
sequentialization (i.e. the number of variables in the sequentialization) as directly proportional to the number of auxiliary variables that are required to build a compositional proof. Concurrent programs that are "loosely coupled" often require only a small number of auxiliary variables to be exposed, and hence admit efficient sequentializations.

We also describe our experience in applying our sequentialization to prove a suite of concurrent programs entirely correct by using deductive verification of their sequentializations. More precisely, we wrote rely-guarantee proof annotations for some concurrent programs by formulating the rely and guarantee conditions, the loop invariants, and pre- and post-conditions for every function. We then sequentialized the concurrent program and also transformed the rely-guarantee proof annotations to corresponding proof annotations on the sequential program. As we show, in this translation, rely and guarantee conditions naturally get transformed to pre- and post-conditions of methods, while loop-invariants and preand post-conditions get translated to loop invariants and pre- and post-conditions in the sequential program. Then, using an automatic sequential program verifier BoogIE, we verified the sequentializations correct. BooGIE takes our programs with the proof annotations, generates verification conditions, and discharges them using an automatic theorem prover (SMT solver).

The above use of sequentialization for deductive verification is not the best use of our sequentializations, as given rely-guarantee proofs, simpler techniques for statically verifying them are known 6]. However, our sequentializations can be applied even when the rely-guarantee proofs are not known, provided the sequential verification tool is powerful to prove it correct. Indeed, we have also used the sequentialization followed by an automatic predicate-abstraction tool (SLAM [2]) to prove some concurrent programs correct.

In summary, the result presented in this paper shows a surprising connection between compositional proofs and sequentializability. We believe that this constitutes a fundamental theoretical understanding of when concurrent programs are efficiently sequentializable, and offers the first efficient sequentializations that work without underapproximation restrictions, enabling us to verify concurrent program entirely using sequentializations.

Related work: Thread-modular verification [6]7 is in fact precisely the same as compositional verification á la Jones, but has been adapted to both modelchecking [7] and extended static checking [6]. Our result can be hence seen as showing how thread-modular verification of concurrent programs can be reduced to pure sequential verification. There has also been work on using counterexample guided predicate-abstraction and refinement for rely-guarantee reasoning [4], and building rely-guarantee interfaces using learning [3].

\section{A Compositional Abstract Semantics for Programs}

We define a non-standard compositional semantics for concurrent programs, different from the traditional semantics, in order to capture when a parallel composition of programs can be argued compositionally to satisfy a specification. This 
semantics is parameterized by a set of auxiliary variables, and is the semantic analog of compositional rely-guarantee proofs pioneered by Jones [9].

Let us fix two processes $P_{1}$ and $P_{2}$, working concurrently, with local variables $L_{1}$ and $L_{2}$ respectively, and a set of shared variables $S$ (assume $L_{1}, L_{2}$ and $S$ are pairwise disjoint, without loss of generality). For any set of (typed) variables $V$, let $\mathrm{Val}_{V}$ denote the set of valuations of $V$ to their respective data-domains (data-domains are finite or countably infinite). For any $u \in V a l_{V}$, let $u \downarrow V^{\prime}$ denote the valuation $u$ restricted to the variables in $V \cap V^{\prime}$. We extend this notation to sets of valuations, $U \downarrow V^{\prime}$. Also, for any $u \in V a l_{V}$ and $u^{\prime} \in V a l_{V^{\prime}}$, where $V \cap V^{\prime}=\emptyset$, let $u \cup u^{\prime}$ denote the unique valuation in $V a l_{V \cup V^{\prime}}$ that extends $u$ and $u^{\prime}$ to $V \cup V^{\prime}$.

Let Init $\subseteq\left(V a l_{L_{1}} \times V a l_{L_{2}} \times V a l_{S}\right)$ be the set of initial global configurations of $P_{1} \| P_{2}$. Let $\delta_{1} \subseteq\left(\operatorname{Val}_{L_{1}} \times \operatorname{Val}_{S} \times \operatorname{Val}_{L_{1}} \times V a l_{S}\right)$ and $\delta_{2} \subseteq\left(\operatorname{Val}_{L_{2}} \times \operatorname{Val}_{S} \times \operatorname{Val}_{L_{2}} \times\right.$ $\mathrm{Val}_{S}$ ) be the local transition relations of $P_{1}$ and $P_{2}$, respectively.

The natural (interleaving) semantics of $P_{1} \| P_{2}$ is, of course, defined by the function $\delta \subseteq\left(\operatorname{Val}_{L_{1}} \times V a l_{L_{2}} \times V a l_{S} \times V a l_{L_{1}} \times \operatorname{Val}_{L_{2}} \times \operatorname{Val}_{S}\right)$, where $\delta\left(l_{1}, l_{2}, s, l_{1}^{\prime}, l_{2}^{\prime}, s^{\prime}\right)$ holds iff $\delta_{1}\left(l_{1}, s, l_{1}^{\prime}, s^{\prime}\right)$ holds and $l_{2}^{\prime}=l_{2}$, or $\delta_{2}\left(l_{2}, s, l_{2}^{\prime}, s^{\prime}\right)$ holds and $l_{1}^{\prime}=l_{1}$. The set of reachable states according to this relation, Reach, is defined as the set of global states that can be reached from the initial state.

Let us now define the non-standard compositional semantics of $P_{1} \| P_{2}$. This definition is parameterized by a set of auxiliary variables $A \subseteq L_{1} \cup L_{2}$.

Definition 1. The semantics of the compositional semantics of parallel composition with respect to the set of auxiliary variables $A$, denoted $P_{1} \|_{A} P_{2}$, is defined using the four sets:

$$
\begin{gathered}
R_{1} \subseteq\left(\operatorname{Val}_{L_{1}} \times \operatorname{Val}_{S} \times \operatorname{Val}_{A \cap L_{2}}\right), \\
R_{2} \subseteq\left(\operatorname{Val}_{L_{2}} \times \operatorname{Val}_{S} \times \operatorname{Val}_{A \cap L_{1}}\right), \\
\operatorname{Guar}_{1}, \text { Guar }_{2} \subseteq\left(\operatorname{Val}_{S} \times \operatorname{Val}_{A} \times \operatorname{Val}_{S} \times \operatorname{Val}_{A}\right),
\end{gathered}
$$

which are defined as the least sets that satisfy the following conditions:

\section{a) Initialization:}

- $R_{1}$ contains the set $\left\{\left(l_{1}, s, t\right) \mid l_{1} \cup s \cup t \in \operatorname{Init} \downarrow\left(L_{1} \cup A \cup S\right)\right\}$.

- $R_{2}$ contains the set $\left\{\left(l_{2}, s, t\right) \mid l_{2} \cup s \cup t \in \operatorname{Init} \downarrow\left(L_{2} \cup A \cup S\right)\right\}$.

b) Transitions of $P_{1}:$ If $\left(l_{1}, s, t\right) \in R_{1}$ and $\delta_{1}\left(l_{1}, s, l_{1}^{\prime}, s^{\prime}\right)$ holds, then

- Local update: $\left(l_{1}^{\prime}, s^{\prime}, t\right) \in R_{1}$.

- Update to guarantee: $\left(s, l_{1} \downarrow A \cup t, s^{\prime}, l_{1}^{\prime} \downarrow A \cup t\right) \in$ Guar $_{1}$.

c) Transitions of $P_{2}:$ If $\left(l_{2}, s, t\right) \in R_{2}$ and $\delta_{2}\left(l_{2}, s, l_{2}^{\prime}, s^{\prime}\right)$ holds, then

- Local update: $\left(l_{2}^{\prime}, s^{\prime}, t\right) \in R_{2}$

- Update to guarantee: $\left(s, l_{2} \downarrow A \cup t, s^{\prime}, l_{2}^{\prime} \downarrow A \cup t\right) \in \mathrm{Guar}_{2}$.

d) Interference:

- If $\left(l_{1}, s, t\right) \in R_{1}$ and $\left(s, l_{1} \downarrow A \cup t, s^{\prime}, t^{\prime}\right) \in$ Guar $_{2}$, then $\left(l_{1}, s^{\prime}, t^{\prime} \downarrow L_{2}\right) \in R_{1}$.

- If $\left(l_{2}, s, t\right) \in R_{2}$ and $\left(s, l_{2} \downarrow A \cup t, s^{\prime}, t^{\prime}\right) \in \operatorname{Guar}_{1}$, then $\left(l_{2}, s^{\prime}, t^{\prime} \downarrow L_{1}\right) \in R_{2}$.

The set of reachable states according to the non-standard compositional semantics with respect to the set of auxiliary variables $A$ is defined as

$$
\operatorname{Reach}_{A}=\left\{\left(l_{1}, s, l_{2}\right) \mid\left(l_{1}, s, l_{2} \downarrow A\right) \in R_{1} \text { and }\left(l_{2}, s, l_{1} \downarrow A\right) \in R_{2}\right\} .
$$


Intuitively, under the compositional semantics, we track independently the view of $P_{1}$ (and $P_{2}$ ) using valuations of its local variables, shared variables, and the subset of the other process's local variables declared to be auxiliary (using the sets $R_{1}$ and $R_{2}$ ). Furthermore, we keep the set of guarantee transition-relations Guar $_{1}$ and Guar $_{2}$ that summarize what transitions $P_{1}$ and $P_{2}$ can take, but restricted to the auxiliary and shared variables only. The guarantee-relation of $P_{1}$ is used to update the view of $P_{2}$ (i.e. $R_{2}$ ), and vice versa. The crucial aspect of the definition above is that it ignores the correlation between local variables of $P_{1}$ and $P_{2}$ that are not defined to be auxiliary variables. The computation of $P_{1} \|_{A} P_{2}$ hence proceeds mostly locally, with updates using the guarantee relation of the other process (which affects shared and auxiliary variables only), and is combined in the end to get the set of globally reachable configurations.

It is not hard to see that Reach $\subseteq \operatorname{Reach}_{A}$, for any $A$. Hence, the compositional semantics is an over-approximation of the set of reachable states of the program, and proving that a program is safe under the compositional semantics is sufficient to prove that the program is safe. Moreover, when the auxiliary variables include all local variables (including the program counter and local call stack), the compositional semantics coincides with the natural semantics.

The above definitions and rules can be generalized to $k$ processes running in parallel, and we can define the compositional semantics $P_{1}\left\|_{A} P_{2}\right\|_{A} \ldots \|_{A} P_{n}$ where $A$ is subset of local variables of each process.

\section{The rely-guarantee proof method of Jones}

The rely-guarantee method of Jones [9] essentially builds compositional relyguarantee proofs using a similar abstraction. Given sequential programs $P_{1}$ and $P_{2}$, and a pre-condition pre and a post-condition post for $P_{1} \| P_{2}$, the relyguarantee proof technique over a set of auxiliary variables $A$ involves providing a pair of tuples, $\left(\right.$ pre $_{1}$, post $_{1}$, rely $_{1}$, guar $\left._{1}\right)$ and $\left(\right.$ pre $_{2}$, post $_{2}$, rely $_{2}$, guar $\left._{2}\right)$, where pre $_{1}$, post $_{1}$, pre $_{2}$, and post pre $_{2}$ anary predicates defining subsets of states, and rely , $_{\text {, }}$ guar $_{1}$, rely $_{2}$, guar $_{2}$ are binary relations defining transformations of the shared variables and the auxiliary variables $A$. The meaning of the tuple for $P_{1}$ is that, when $P_{1}$ is started with a state satisfying pre $_{1}$ and in an environment that could change the auxiliary variables and shared variables allowed by rely,$P_{1}$ would make transitions that accord to guar $_{1}$, and if it terminates, will satisfy post at $_{1}$ the exit. An analogous meaning holds for $P_{2}$. Note that rely , rely $_{2}$, guar $_{1}$ and guar $_{2}$ are defined over shared variables and the auxiliary variables. The programs $P_{1}$ and $P_{2}$ are proved to satisfy these conditions using a local proof by considering each $P_{i}$ interacting with a general environment satisfying rely; in particular invariants of $P_{i}$ needed to establish the Hoare-style proof of $P_{i}$ should be invariant or stable with respect to rely .

The following proof rule can then be used to prove partial correctness of $P_{1} \| P_{2}$ :

$$
\begin{aligned}
& \text { guar }_{1} \Rightarrow \text { rely }_{2}, \quad \text { guar }_{2} \Rightarrow \text { rely }_{1}, \\
& P \models\left(\text { pre } \text { post }_{1}, \text { rely }_{1}, \text { guar }_{1}\right), \quad Q \models\left(\text { pre }_{\text {post }}, \text { rely }_{2}, \text { guar }_{2}\right)
\end{aligned}
$$

$$
P \| Q \models\left(\text { pre, } \text { post }_{1} \wedge \text { post }_{2}\right)
$$


The rely-guarantee method works also for nested parallellism compositionally; see 916] for details.

It is easy to see that a compositional rely-guarantee proof of $P_{1} \| P_{2}$, over a set of auxiliary variables $A$, is really a proof that the compositional semantics of $P_{1} \|_{A} P_{2}$ is correct. Note that if $P_{1} \|_{A} P_{2}$ is correct, it does not imply a relyguarantee proof exists, however, as proofs have limitations of the logical syntax used to write the rely and guarantee conditions, and hence do not always exist.

\section{The main result}

We can now state the main result of this paper. We show that, given a parallel composition of sequential programs $P_{1}|| P_{2}\|\ldots\| P_{n}$ with assertions, and a set of auxiliary variables $A$, we can build a sequential program $S$ with assertions such that $S$ has the following properties:

- At any point, the scope of $S$ contains at most one copy of the local variables of a single process $P_{i}$, three copies of the auxiliary variables, and at most three copies of the shared variables.

- The compositional semantics of $P_{1}\left\|_{A} P_{2}\right\|_{A} \ldots \|_{A} P_{n}$ with respect to the auxiliary variables $A$ satisfies its assertions iff $S$ satisfies its assertions.

- If $S$ satisfies its assertions, then $P_{1}\left\|P_{2}\right\| \ldots \| P_{n}$ also satisfies its assertions.

The first remark above says that the sequentialized program has less variables in scope than the naive product of the individual processes; the sequentialization intuitively simulates the processes separately, keeping track of only an extra copy of auxiliary variables and shared variables. Second, the sequentialization is a precise reduction of the verification problem, provided the concurrent program can be proved compositionally (i.e. if the auxiliary variables are sufficient to make the compositional semantics of the program be assertion-failure free). Finally, the sequential program is an over-approximation of the behaviors of the parallel program for any set of auxiliary variables, and hence proving it correct proves the parallel program correct.

The above result will be formalized in the sequel (see Theorem 1) for a class of parallel programs that has sequential recursive functions, but with no thread creation or dynamic memory allocation (the result can be extended to dynamic data-structures but will require mechanisms to cache heap-structures and compare them for equality). Our main theorem hence states that any parallel program that is amenable to compositional reasoning can be sequentialized, where the number of new variables added in the sequentialization grows with the number of auxiliary variables required to prove the program correct. We utilize the sequentialization result in one verification context, namely deductive verification, to build a compositional deductive verification tool for concurrent programs using the sequential verifier BooGIE.

\section{A High Level Overview of the Sequentialization}

In this section, we give a brief overview of our sequentialization. For ease of explanation, let us consider a concurrent program consisting of two processes $P_{1}$ 
and $P_{2}$. Let $A$ be the set of auxiliary variables and assume that the compositional semantics of the concurrent program is correct with respect to $A$.

Assume we had functions $G_{1}\left(s^{*}, a^{*}\right)$ (and $G_{2}\left(s^{*}, a^{*}\right)$ ) that somehow takes a shared and auxiliary state $\left(s^{*}, a^{*}\right)$ and non-deterministically returns all states $(s, a)$ such that $\left(s^{*}, a^{*}, s, a\right) \in$ Guar $_{1}$ (respectively Guar $_{2}$ ), where Guar $_{1}$ and Guar $_{2}$ are as in Definition 1. Then we could write a function that computes the states reachable by $P_{1}$ according to the compositional semantics (i.e. $R_{1}$ in Definition 1) using the following code:

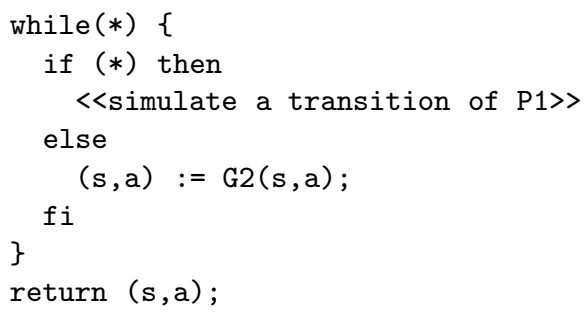

In other words, we could write a sequential program that returns precisely the states in $R_{1}$, by interleaving simulations of $P_{1}$ with calls to $G_{2}$ to compute interference according to Guar $_{2}$ (see Definition 1). We can similarly implement the sequential code that explores $R_{2}$ using calls to $G_{1}$.

Note that on two successive calls to $G_{2}()$, there is no preservation of the local states of $P_{2}$, except its variables declared to be auxiliary. However, we do not have to preserve the exact local state of $P_{2}$ as we are not simulating the natural semantics of the program, but only its compositional semantics with respect to auxiliary variables $A$. This is the crux of the argument as to why we can sequentially compute $R_{1}$ without simultaneously tracking all the local variables of $P_{2}$.

Now, turning to the function $G_{2}$ (and $G_{1}$ ), consider Definition 1 again, and notice that, given $\left(s^{*}, a^{*}\right)$, in order to compute $(s, a)$ such that $\left(s^{*}, a^{*}, s, a\right) \in$ $\operatorname{Guar}_{2}$, we must essentially be able to find a local state $l_{2}$ such that $\left(l_{2}, s^{*}, a^{*} \downarrow\right.$ $\left.L_{1}\right) \in R_{2}$ where $l_{2} \downarrow A=a^{*} \downarrow L_{2}$, and then we can take its transitive closure with respect to $\delta_{2}$. We can hence write $G_{2}$ using the following sequential code:

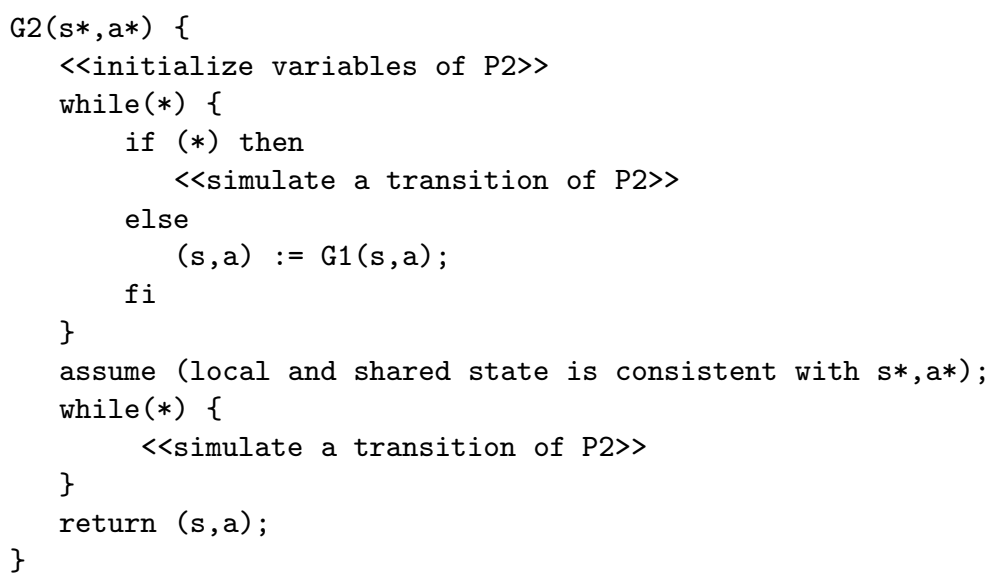


Intuitively, $G_{2}$ starts with the initial state of $P_{2}$ and sets about recomputing a state $\left(l_{2}, s_{2}\right)$ that is compatible with its given input $\left(s^{*}, a^{*}\right)$ (i.e. with $s_{2}=s^{*}$ and $\left.l_{2} \downarrow A=a^{*} \downarrow L_{2}\right)$. It does this by essentially running the code for $R_{2}$ (i.e. by simulating $P_{2}$ and calling $G_{1}$ ). Once it has found such a state, it simulates $P_{2}$ for a while longer, and returns the resulting state.

We hence get four procedures that compute $R_{1}, R_{2}$, Guar $_{1}$ and Guar $_{2}$, respectively, with mutually recursive calls between the functions computing Guar $_{1}$ and Guar $_{2}$. The correctness of the sequential programs follow readily from Definition 1, as it is a direct encoding of that computation. Our sequentialization transformation essentially creates these functions $G_{1}$ and $G_{2}$. However, since our program cannot have statements like "simulate a transition of P2", we perform a syntactic transformation of the concurrent code into a sequential code, where control code is inserted between statements of the concurrent program in order to define the functions $G_{1}$ and $G_{2}$. This complication combined with the handling of recursive functions makes the translation quite involved; however, the above explanation captures the crux of the construction.

\section{Sequential and Concurrent Programs}

Our language for concurrent programs consists of a parallel composition of recursive sequential programs. Variables in our programs are defined over integer and Boolean domains. The syntax of programs is defined by the following grammar:

$$
\begin{aligned}
& \langle\text { conc-pgm }\rangle::=\langle\text { decl }\rangle^{*}\langle\text { pgm-list }\rangle \\
& \langle\text { pgm-list }\rangle \quad::=\langle\text { pgm-list }\rangle \|\langle\text { pgm-list }\rangle \mid\langle p g m\rangle \\
& \langle p g m\rangle \quad::=\langle\text { decl }\rangle^{*}\langle\text { proc }\rangle^{*} \\
& \langle\text { proc }\rangle \quad::=f(\bar{x}) \text { begin }\langle\text { decl }\rangle^{*}\langle\text { stmt }\rangle \text { end } \\
& \langle s t m t\rangle \quad::=\langle s t m t\rangle ;\langle s t m t\rangle|\operatorname{skip}| \bar{x}:=\operatorname{expr}(\bar{x})|\bar{x}:=f(\bar{y})| \\
& f(\bar{x})|\operatorname{return} \bar{x}| \text { assume } b \text {-expr | assert b-expr | } \\
& \text { if } b \text {-expr then }\langle s t m t\rangle \text { else }\langle s t m t\rangle \text { fi | } \\
& \text { while b-expr do }\langle\text { stmt }\rangle \text { od | atomic }\{\langle s t m t\rangle\} \\
& \langle\text { decl }\rangle \quad:=\text { int }\langle\text { var-list }\rangle ; \quad \text { bool }\langle\text { var-list }\rangle \text {; } \\
& \langle\text { var-list }\rangle \quad::=\langle\text { var-list }\rangle,\langle\text { var-list }\rangle \mid\langle\text { literal }\rangle
\end{aligned}
$$

A concurrent program consists of $k$ sequential program components $P_{1} \ldots P_{k}$ (for some $k$ ) communicating with each other through shared variables $\mathcal{S}$. These shared variables are declared in the beginning of the concurrent program (we assume integer variables are initialized to 0 and Boolean variables to false). Each sequential component consists of a procedure called main and a list of other procedures. The control flow for all sequential components $P_{i}$ starts in the corresponding main procedure, which we call main $_{i}$. The main for all sequential components has zero arguments and no return value.

Each procedure is a declaration of local variables followed by a sequence of statements, where statements can be simultaneous assignments, function calls 
(call-by-value) that take in multiple parameters and return multiple values, conditionals, while loops, assumes, asserts, atomic, and return statements. In the above syntax, $\bar{x}$ represents a vector of variables. We allow non-determinism in our programs; boolean constants are true, false and $*$, where $*$ evaluates nondeterministically to true or false.

The safety specifications for both concurrent and sequential programs are expressed in our language as assert statements. The semantics of an assume statement is slightly different. If the value of the boolean expression (b-expr) evaluates to true, then the assume behaves like a skip. Otherwise, if the boolean expression evaluates to false, the program silently terminates. Synchronization and atomicity are achieved by the atomic construct. All the statements enclosed in the atomic block are executed without any interference by the other processes. Locks can be simulated in our syntax by modeling a lock $l$ as an integer variable 1 and by modeling $P_{i}$ acquiring $l$ using the code:

$$
\text { atomic }\{\text { assume }(1=0) ; 1:=i ;\}
$$

and modeling the release with the code:

$$
\text { atomic }\{\text { if }(1=i) \text { then } 1:=0 ;\}
$$

We assume programs do not have nested atomic blocks.

The syntax of sequential programs is the same as the syntax of concurrent programs except that we disallow the parallel composition operator $(\|)$ and the atomic construct.

\section{The Sequentialization}

In this section, we describe our sequentialization for concurrent programs and argue its correctness.

Let us fix a concurrent program with shared variables $S$ and auxiliary variables $A$; we assume auxiliary variables are global in each thread $P_{i}$. Let the concurrent program be composed of $k$ sequential components.

The sequential program corresponding to the concurrent program will have a new function main, and additionally, as explained in Section [3, will have a procedure $G_{i}$ for each sequential component $P_{i}$ of the concurrent program that semantically captures the guarantee Guar $_{i}$ of $P_{i}$. The procedure $G_{i}$ takes a shared state $\left(\overline{s^{*}}\right)$ and auxiliary state $\left(\overline{a^{*}}\right)$ as input and returns $(\bar{s}, \bar{a})$ such that $\left(\overline{s^{*}}, \overline{a^{*}}, \bar{s}, \bar{a}\right) \in$ Guar $_{i}$. Finally, each $G_{i}()$ is formed using procedures that are obtained by transforming the process $P_{i}$ (using the function $\tau_{i}$ [] shown below that essentially inserts the interference code $\mathcal{I}_{i}$ shown in Figure 1 between the statements of $P_{i}$ ).

The shared variables and auxiliary variables are modeled as global variables in the sequential program. Furthermore, we have an extra copy of the shared and auxiliary variables $\left(\overline{s^{*}}\right.$ and $\left.\overline{a^{*}}\right)$ that are used to pass shared and auxiliary states between the processes $G_{i}()$. We also have a copy of shared and auxiliary variables $\left(\overline{s^{\prime}}\right.$ and $\left.\overline{a^{\prime}}\right)$ that are declared to be local in each procedure to store 
a shared and auxiliary state and restore it after a call to a function $G_{j}()$ to compute interference. Besides these, the sequential program also uses global Boolean variables $z$ and term; intuitively, $z$ is used to keep track of when the shared and auxiliary state $\overline{s^{*}}$ and $\overline{a^{*}}$ has been reached and term (for terminate) is used to signal that $G_{i}()$ has finished computing and wants to return the value.

- Global variable declarations are:

// insert declaration for $\overline{\mathrm{s}}, \overline{\mathrm{a}}, \overline{\mathrm{s}^{*}}, \overline{\mathrm{a}^{*}}$ as global variables decl bool term, $\mathbf{z}$;

- The function main() is defined as: main() begin G_1() end

- Each function $G_{i}()$ is defined as below:

G_i() begin

$z:=$ false; term := false;

$\overline{\mathrm{s}^{*}}:=\overline{\mathrm{s}} ; \quad \overline{\mathrm{a}^{*}}:=\overline{\mathrm{a}}$;

// insert code to initialize $\overline{\mathrm{s}}, \overline{\mathrm{a}}$

main_i();

assume (term = true);

return

end

- The function $\tau_{i}$ that transforms the program for $P_{i}$ is defined as:

- $\tau_{i}[\mathrm{f}(\overline{\mathrm{x}})$ begin decl stmt end $]=$

$\mathrm{f}(\overline{\mathrm{x}})$ begin decl

// insert declaration of $\overline{\mathrm{s}^{\prime}}, \overline{\mathrm{a}^{\prime}}$ as local variables.

end

- $\tau_{i}\left[\mathcal{S}_{1} ; \mathcal{S}_{2}\right]=\tau_{i}\left[\mathcal{S}_{1}\right] ; \tau_{i}\left[\mathcal{S}_{2}\right]$

- $\tau_{i}[\mathcal{S}]=\mathcal{I}_{i} ; \mathcal{S}$ where $\mathcal{S}$ is an assignment, skip, assume, assert, a function call or a return statement.

- $\tau_{i}[$ while b-expr do $\mathcal{S}$ od $]=\mathcal{I}_{i}$; while b-expr do $\tau_{i}[\mathcal{S}] ; \mathcal{I}_{i}$ od

- $\tau_{i}\left[\right.$ if $b-\operatorname{expr}$ then $\mathcal{S}_{1}$ else $\mathcal{S}_{2}$ fi] $=$

$\mathcal{I}_{i}$; if b-expr then $\tau_{i}\left[\mathcal{S}_{1}\right]$ else $\tau_{i}\left[\mathcal{S}_{2}\right]$ fi

- $\tau_{i}[$ atomic $\{\mathcal{S}\}]=\mathcal{I}_{i} ; \mathcal{S}$

The procedure main in the sequential program simply calls the method $G_{1}$.

The procedure $G_{i}$ is obtained from the corresponding program component $P_{i}$ by a simple transformation. At a high level, this procedure first copies the incoming shared and auxiliary state into the variables $\overline{s^{*}}$ and $\overline{a^{*}}$. It then computes a local state of $P_{i}$ which is consistent with the state $\left(\overline{s^{*}}, \overline{a^{*}}\right)$ (at which point $z$ turns to true), and then non-deterministically simulates the transitions of $P_{i}$ from this local state, to return a reachable shared state $\bar{s}$ and auxiliary state $\bar{a}$. Every time $G_{i}$ is called, it starts from its initial state, and simulates $P_{i}$, interleaving it with the control code $\mathcal{I}_{i}$ given in Figure 1

The interference code $\mathcal{I}_{i}$ (Figure 1) keeps track of whether the incoming state $\left(\overline{s^{*}}, \overline{a^{*}}\right)$ has been reached through a boolean variable $z$ which is initialized to 
false. If $z$ is false (i.e. the state $\left(\overline{s^{*}}, \overline{a^{*}}\right)$ has not been reached), then before any transition of $P_{i}$, the control code can non-determinsitically choose to invoke its environment (in doing so, in order to preserve its input $\overline{s^{*}}, \overline{a^{*}}$, it stores them in a local state and restores them after the call returns and restores its variables $z$ and term to false).

When the state $\left(\overline{s^{*}}, \overline{a^{*}}\right)$ is reached, $z$ can be non-deterministically set to true, from which point no interference code $G_{j}$ can be called, and only local computation proceeds, till non-deterministically the program decides to terminate by setting term to true. Once term is true, the code pops the controlstack all the way back to reach the function $G_{i}$ which then returns to its caller, returning the new state in $(\bar{s}, \bar{a})$. Note that the state $z=$ false, term $=$ false corresponds to the first while loop in the code for the guarantee $G_{2}$ in Section 3. Similarly, setting term to true corresponds to the termination of the second while loop. We conclude this section by stating our main theorem:

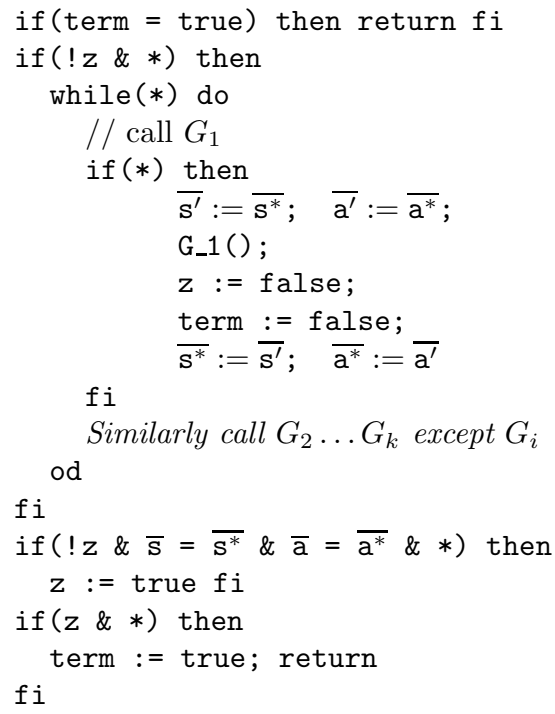

Fig. 1. The interference control code $\mathcal{I}_{i}$

Theorem 1. Let $C$ be a concurrent program with auxiliary variables $A$ (assumed global), and let $S_{C, A}$ be its sequentialization with respect to $A$. Then the compositional semantics of $C$ with respect to the auxiliary variables $A$ has no reachable state violating any of its assertions iff $S_{C, A}$ violates none of its assertions.

\section{Illustration of a sequentialization}

Figure 2 shows a concurrent program consisting of two threads, say $P_{1}$ and $P_{2}$. The program consists of a shared variable $x$ whose initial value is zero. Both the threads atomically increment the value of $x$. Let $A=\{p c 1, p c 2\}$ be the auxiliary variables capturing the control position in the respective processes and let the initial value of these variables also be zero. In general, new auxiliary variables may be needed for performing compositional proofs; these new variables are written to but never read from in the program, and hence do not affect the semantics of the original program; see 915 .

It can be easily seen that the compositional semantics of this program with respect to the auxiliary variables $A$ is correct. Figure 3 shows the sequential program obtained from the sequentialization of this concurrent program with respect to these auxiliary variables. Our result allows us to verify the concurrent program in Figure 2 by verifying the correctness of its sequentialization with respect to the auxiliary variables $A$, shown in Figure 3 . 


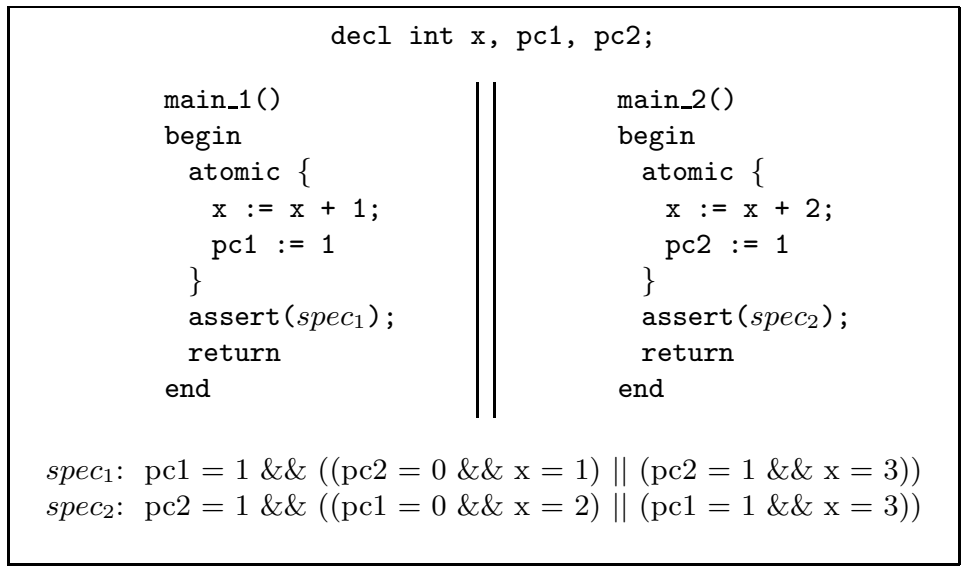

Fig. 2. An example program

\begin{tabular}{|c|c|c|}
\hline 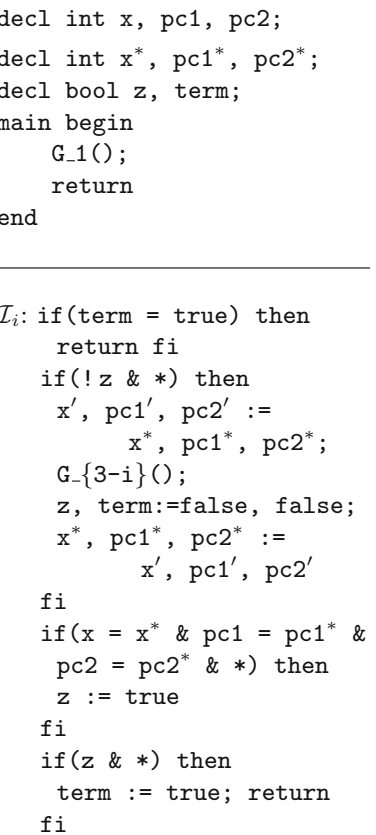 & 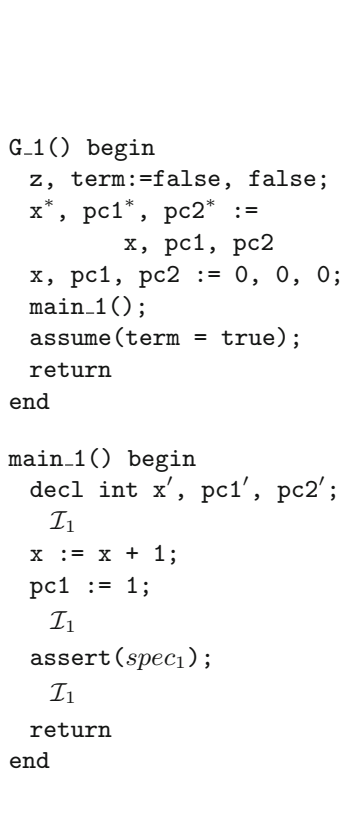 & 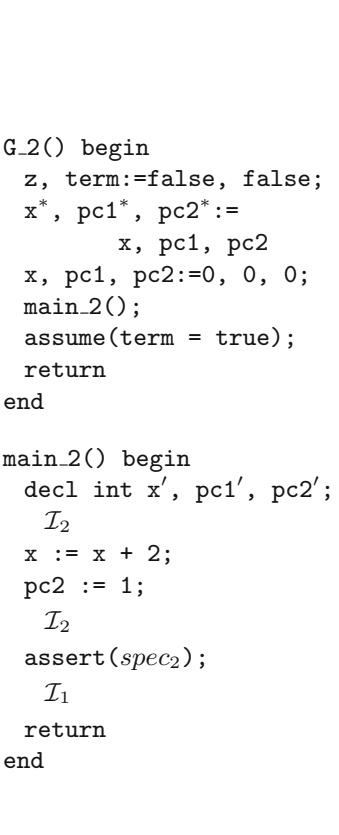 \\
\hline
\end{tabular}

Fig. 3. The sequential program obtained from the concurrent program in Figure 2

\section{Experience}

The sequentialization used in this paper can be used to verify a concurrent program using any sequential verification tool. This includes tools based on 
abstract interpretation and predicate abestraction, those based on bounded model-checking, as well as those based on deductive-verification based extended static checking.

Deductive verification: We used the sequentialization for proving concurrent programs using deductive verification. Given a concurrent program and its Jonesstyle rely-guarantee proof annotations (pre, post, rely, guar, and loop-invariants), we sequentialized it with respect to the auxiliary variables and syntactically transformed the user-provided proof annotations to obtain the proof annotations (pre-conditions and post-conditions of methods and loop invariants) of the sequential program. In general, the pre-condition of method $G_{i}$ asserts that "term = false" and its post-condition asserts that the guarantee guar $_{i}$ is true across the function (if term is true). Furthermore, the pre-conditions and postconditions of every function in $P_{i}$ gets translated to pre-conditions and postconditions in its sequentialization with the extra condition that guar $_{i}$ is true when term is equal to true.

These annotated sequential programs were fed to the sequential verifier BooGIE that generates verification conditions that are in turn solved by an SMT solver (Z3 in this case). If the sequential program is proved correct, it proves the correctness of the original concurrent program. Note that though similar static extended checking techniques are known for the rely-guarantee method [6], our technique allows one to use just a sequential verifier like BoogIE to prove the program correct, and requires no other decision problems to be solved (the checking in [6], for example, requires a separate call to a theorem prover to check guarantees are reflexive and transitive, etc.)

We used this technique to prove correct the following set of concurrent programs: X++ (Figure 2), Lock [7], Peterson's mutual exclusion algorithm, the Bakery protocol, ArrayIndexSearch [9], GCD [5], and a simplified version of a Windows NT Bluetooth driver. Lock is a simple example program consisting of two threads that modify a shared variable after acquiring a lock; the safety condition in the example asserts that these modifications cannot occur concurrently. The program ArrayIndexSearch finds the least index of an array such that the value at that index satisfies a given predicate, and consists of two threads, one that searches odd indices and the other that searches even indices, and communicate on a shared variable index that is always kept updated to the current least index value. GCD is a concurrent version of Euclid's algorithm for computing the greatest common divisor of any two numbers; here the two concurrent threads update the pair of integers.

The Windows NT bluetooth driver is a parameterized program (i.e. has an unbounded number of threads). It consists of two types of threads: there is one stopper-thread and an unbounded number of adder-threads. A stopper calls a procedure to halt the driver, while an adder calls a procedure to perform $\mathrm{I} / \mathrm{O}$ in the driver. The $\mathrm{I} / \mathrm{O}$ is successfully handled if the driver is not stopped while it executes. The program, though small, has an intricate global invariant that requires a shared variable to reflect the number of active adder threads. 
Table 1. Experimental Results. Evaluated on Intel dual-core 1.6GHz, 1Gb RAM.

\begin{tabular}{||c||c|c|c||c|c||c||}
\hline \multicolumn{1}{||c||}{} & \multicolumn{3}{c|}{ Concurrent pgm } & \multicolumn{2}{c||}{ Sequential pgm } & \\
\hline Programs & \#Threads & \#LOC & $\begin{array}{c}\text { \#Lines of } \\
\text { annotations }\end{array}$ & \#LOC & $\begin{array}{c}\text { \#Lines of } \\
\text { annotations }\end{array}$ & Time \\
\hline \hline X++ & 2 & 38 & 5 & 113 & 6 & $8 \mathrm{~s}$ \\
\hline Lock & 2 & 50 & 9 & 184 & 10 & $122 \mathrm{~s}$ \\
\hline Peterson & 2 & 52 & 35 & 232 & 36 & $145 \mathrm{~s}$ \\
\hline Bakery & 2 & 55 & 8 & 147 & 13 & $18 \mathrm{~s}$ \\
\hline ArrayIndexSearch & 2 & 74 & 17 & 222 & 21 & $126 \mathrm{~s}$ \\
\hline GCD & 2 & 78 & 23 & 279 & 29 & $869 \mathrm{~s}$ \\
\hline Bluetooth & unbdd & 69 & 20 & 276 & 55 & $107 \mathrm{~s}$ \\
\hline
\end{tabular}

Table 1 gives the experimental result:1. For each program, we report the number of threads in the concurrent program, the number of lines of code in the concurrent program and its sequentialization, the number of lines of annotations in both the concurrent program (which includes rely/guarantee annotations and loop invariants) and its sequentialization, and the time taken by BoogIE to verify the sequentialized program.

BoogIE was able to verify the correctness of all our programs. All these programs except the Windows NT bluetooth driver consist of two threads and are sequentialized as detailed in Section 5. The Bluetooth driver is an example of a parameterized program running any number of instances of the adder threads. In our sequentialization, we model the environment consisting of all the adder threads together with a single procedure. If we keep track of the number of adders at a particular program location (counter abstraction 14]) and expose these auxiliary variables, it turns out that the device driver can be proved correct under compositional semantics. We used this rely-guarantee proof, sequentialized the program, and used BoogiE to prove the Bluetooth driver correct in its full generality.

Predicate abstraction: We have also used our sequentialization followed by the predicate-abstraction tool SLAM [2] to prove programs automatically correct. In this case, we need no annotations and just the set of auxiliary variables. We were able to automatically prove the correctness of the programs $\mathrm{X}++$, Lock, Peterson and the Bakery protocol, in negligible time.

\section{$7 \quad$ Future Directions}

One drawback of our sequentialization is that it creates recursive programs, even when the concurrent program has no recursion. It is hence natural to ask whether there is a sequentialization that does not introduce recursion. We know indeed of such a sequentialization, where the process $G_{i}$ does not start from the initial state to match the state given to it, but rather "jumps" directly to a state consistent with the state given to it. This, of course, does not capture compositional semantic

\footnotetext{
${ }^{1}$ Experiments available at http://www.cs .uiuc.edu/\$\sim\$garg11/tacas11
} 
reasoning precisely, and seems to be an over-approximation of it. Evaluating the effectiveness of this translation is an interesting future direction.

Finally, since our sequentialization captures the concurrent program without under-approximations, utilizing the sequentialization followed by techniques such as static analysis, predicate-abstraction, and even bounded model-checking, would be interesting directions to pursue.

Acknowledgements. This work is partially supported by NSF grants \#0747041 and \#1018182.

\section{References}

1. Alur, R., Madhusudan, P., Nam, W.: Symbolic compositional verification by learning assumptions. In: Etessami, K., Rajamani, S.K. (eds.) CAV 2005. LNCS, vol. 3576, pp. 548-562. Springer, Heidelberg (2005)

2. Ball, T., Rajamani, S.K.: The SLAM project: debugging system software via static analysis. In: POPL, pp. 1-3. ACM, New York (2002)

3. Cobleigh, J.M., Giannakopoulou, D., Păsăreanu, C.S.: Learning assumptions for compositional verification. In: Garavel, H., Hatcliff, J. (eds.) TACAS 2003. LNCS, vol. 2619, pp. 331-346. Springer, Heidelberg (2003)

4. Cohen, A., Namjoshi, K.S.: Local proofs for global safety properties. In: Damm, W., Hermanns, H. (eds.) CAV 2007. LNCS, vol. 4590, pp. 55-67. Springer, Heidelberg (2007)

5. Feng, X.: Local rely-guarantee reasoning. In: POPL, pp. 315-327. ACM, New York (2009)

6. Flanagan, C., Freund, S.N., Qadeer, S.: Thread-modular verification for sharedmemory programs. In: Le Métayer, D. (ed.) ESOP 2002. LNCS, vol. 2305, pp. 262-277. Springer, Heidelberg (2002)

7. Flanagan, C., Qadeer, S.: Thread-modular model checking. In: Ball, T., Rajamani, S.K. (eds.) SPIN 2003. LNCS, vol. 2648, pp. 213-224. Springer, Heidelberg (2003)

8. Ghafari, N., Hu, A.J., Rakamarić, Z.: Context-bounded translations for concurrent software: An empirical evaluation. In: van de Pol, J., Weber, M. (eds.) SPIN 2010. LNCS, vol. 6349, pp. 227-244. Springer, Heidelberg (2010)

9. Jones, C.B.: Tentative steps toward a development method for interfering programs. ACM Trans. Program. Lang. Syst. 5(4), 596-619 (1983)

10. La Torre, S., Madhusudan, P., Parlato, G.: Reducing context-bounded concurrent reachability to sequential reachability. In: Bouajjani, A., Maler, O. (eds.) CAV 2009. LNCS, vol. 5643, pp. 477-492. Springer, Heidelberg (2009)

11. La Torre, S., Madhusudan, P., Parlato, G.: Sequentializing parameterized programs (2010), http://www.cs.uiuc.edu/ madhu/seqparam.pdf

12. Lahiri, S.K., Qadeer, S., Rakamarić, Z.: Static and precise detection of concurrency errors in systems code using SMT solvers. In: Bouajjani, A., Maler, O. (eds.) CAV 2009. LNCS, vol. 5643, pp. 509-524. Springer, Heidelberg (2009)

13. Lal, A., Reps, T.W.: Reducing concurrent analysis under a context bound to sequential analysis. In: Gupta, A., Malik, S. (eds.) CAV 2008. LNCS, vol. 5123, pp. 37-51. Springer, Heidelberg (2008)

14. Lubachevsky, B.D.: An approach to automating the verification of compact parallel coordination programs. Acta Inf. 21, 125-169 (1984)

15. Owicki, S.S., Gries, D.: An axiomatic proof technique for parallel programs. Acta Inf. 6, 319-340 (1976)

16. Xu, Q., de Roever, W.P., He, J.: The rely-guarantee method for verifying shared variable concurrent programs. Formal Asp. Comput. 9(2), 149-174 (1997) 